\title{
Analysis of Deformation of the Human Ear and Canal Caused by Mandibular Movement
}

\author{
Sune Darkner ${ }^{1,2}$, Rasmus Larsen ${ }^{1}$, and Rasmus R. Paulsen ${ }^{2}$ \\ ${ }^{1}$ Department of Informatics and Mathematical Modelling, Technical University of \\ Denmark, Denmark \\ sda@imm.dtu.dk \\ ${ }^{2}$ Oticon A/S, Denmark ${ }^{\star}$
}

\begin{abstract}
Many hearing aid users experience physical discomfort when wearing their device. The main contributor to this problem is believed to be deformation of the ear and ear canal caused by movement of the mandible. Physical discomfort results from added pressure on soft tissue areas in the ear. Identifying features that can predict potential deformation is therefore important for identifying problematic cases in advance. A study on the physical deformation of the human ear and canal due to movement of the mandible is presented. The study is based on laser scannings of 30 pairs of ear impressions from 9 female and 21 male subjects. Two impressions have been taken from each subject, one with open mouth, and one with the mouth closed. All impressions are registered using non-rigid surface registration and a shape model is built. From each pair of impressions a deformation field is generated and propagated to the shape model, enabling the building of a deformation model in the reference frame of the shape model. A relationship between the two models is established, showing that the shape variation can explain approximately $50 \%$ of the variation in the deformation model. An hypothesis test for significance of the deformations for each deformation field reveals that all subjects have significant deformation at Tragus and in the canal. Furthermore, a relation between the magnitude of the deformation and the gender of the subject is demonstrated. The results are successfully validated by comparing the outcome to the anatomy by using a single set of high resolution histological sectionings of the region of interest.
\end{abstract}

\section{Introduction}

A recent survey has shown that physical comfort and acoustical feedback are among the ten most important issues for hearing aid user satisfaction [1]. It is well known among hearing-aid manufacturers that physical deformation of the human ear canal is connected to problems with both comfort and acoustical feedback. Furthermore, it is known that deformation of the ear canal is closely linked to speaking, chewing, yawning, and movement of the mandible in general. The human ear canal consists of a soft and a bony part. The bony part is em-

\footnotetext{
* Thanks to 3D Lab at the department of Orthodontics, Panum Institute, Denmark.
} 


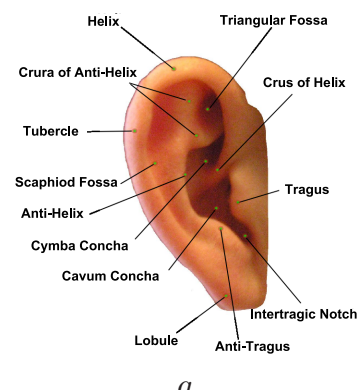

$a$

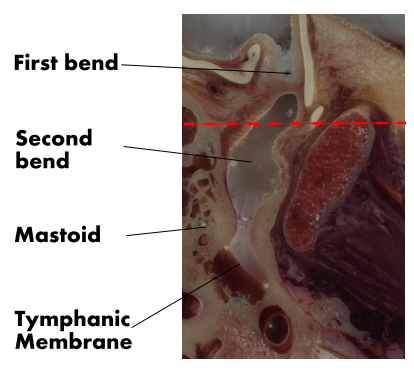

$b$

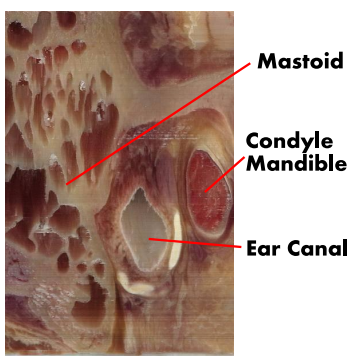

$c$

Fig. 1. (a) Map of the anatomy of the human ear. (b\&c) Histological sectioning of the human ear from 2]. (b) A transversal cut containing the canal. As can be seen, the canal is situated between the mastoid and the mandible before entering the mastoid itself. Furthermore, there are two bends of the canal. The outer bend is called the first bend and the inner, just before the canal enters the mastoid, is called the second bend. (c) A cut in the sagittal plane at the dashed red line of $b$ showing the soft tissue around the ear canal between the mandible and the mastoid.

bedded in the mastoid and, thus, not subject to deformation. However, the soft part of the canal is situated between the mandible and the mastoid surrounded by skin, cartilage, and fat; all tissues that are highly deformable. Fig. 1)(a) shows an anatomical labelling of the human ear. From fig. 1(b) and (c) it is obvious that movement of the mandible will cause deformation of the tissue around it, hence, changing the shape of ear canal. Very little is known about the nature of this deformation seen from a hearing-aid perspective. We believe that systematic knowledge of the shape change of the ear canal can be used in future hearingaid production, thus, creating better and more comfortable hearing aids. In this study, a set of 3D scanned ear impressions (see fig. 2(a)) is used in a non-rigid registration framework to create a shape model and a deformation model. In the following analysis, we try to establish an understanding of where in the ear and canal the significant shape changes occur and if these changes are related to the shape of the ear and canal. Furthermore, it is examined if there is any

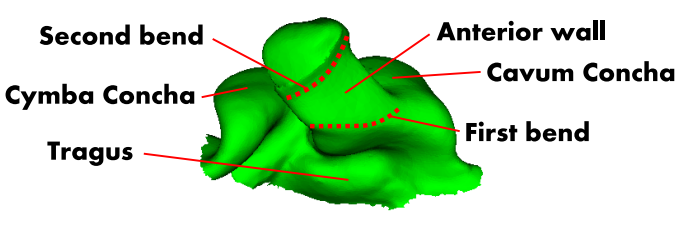

$a$

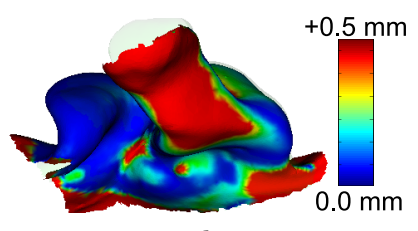

$b$

Fig. 2. (a) A typical scanning of an impression taken from the production. The scanning has been opened at the top, and the lower part has had most artifacts removed manually. Some of the anatomical features have been labelled. (b) The magnitude of the deformation field projected onto the open mouth impression. 
gender-related differences in ear-canal dynamics. All such relations will be beneficial in discovering problematic cases.

\section{Previous Work}

It is only recently that $3 \mathrm{D}$ scanners have been introduced in the production of hearing aids. Therefore, most prior work on ear canal shape was done directly on ear impressions using calipers etc. Oliviera et al. 34 have analyzed the changes that occur in the ear canal due to movement of the mandible and concluded that there is a deformation and also a change in volume. They claim that the deformation only occurs in the coronal plane. However, Grenness et al. [5] have shown that the deformation is more complex, assuming that the Concha is stable during movement of the mandible. This claim remains to be proven. Finally, Pirzanski 6] has analyzed the dynamics of the ear canal with the goal of increasing hearing aid users acceptance rates. However, all of the above is based on manual measurements and manual registration, which is prone to error. A statistical shape model of the static ear canal based on scanned ear impressions and automated registration have been presented by Paulsen et al. 7].

\section{Data}

The data consists of 60 scannings of ear impression taken from 30 individuals, 21 males and 9 females, ranging from 25 to 65 years of age. Two impressions were taken from each, one with open mouth using a mouth prop to create a similar opening angle for all subjects and one with closed mouth. To ensure consistency, all impressions were made by the same audiologist and scanned on a 3D laser scanner by the same operator.

\section{Inspection of the Anatomy}

The images seen in fig. 1(a) and (b) are a part of a high-resolution histological sectioning study by Sørensen et al. [2]. The data set includes the outer ear, making it possible to investigate the physiology of the human ear and canal. As mentioned, the mandible is situated in front of the ear canal between the first and the second bend. It is known that the tissue surrounding the canal is not directly attached to the mandible. When the mandible moves forward a small void is created, which is filled by the surrounding tissue. It is expected that this will cause a deformation of the wall of the ear canal on the anterior side between the first and the second bend. As fig. 1 $(c)$ shows, the posterior and top side of the canal are situated very close to the mastoid, thus, limiting the amount of deformation on this side of the canal. Tragus and Cavum Concha is situated on the soft tissue surrounding the Mandible. In fact, careful examination reveals that the whole outer ear is situated on soft deformable tissue, especially 
the part below Crus of Helix. Hence, Grenness' assumption of a stable Concha seems to be wrong and the ear below Crus of Helix can be expected to move inwards perpendicular to the sagittal plane as the mouth opens. Inspection of the histological data reveals that the best reference for the data in this study is the Cymba Concha. This part of the ear might also be subject to deformation. However, since it is situated on the outside of the mastoid, contrary to the Cavum Concha which is situated just beneath, it is not as likely as other parts of the outer ear to displace and deform.

\section{Model Generation}

To make a consistent data analysis a frame of reference must be established. A fully automatic rigid registration algorithm by Darkner et al. [8], evaluated by Darkner et al. 9], is used to register the Cymba Concha of all impression pairs. A highly constrained non-rigid surface registration is then applied to create the deformation field, see fig. 2 (b). Secondly, to establish dense point to point correspondence across the population the non-rigid surface registration algorithm is applied to all closed mouth impression. Dense point to point correspondence is generated from the resulting registration using the angle weighted normal method by Bærentzen and Aanæs [10] and ray tracing [11. The result is then propagated to the deformation field for correspondence between the deformations and shapes. The non-rigid registration is based on the diffeomorphic warp presented by Cootes and Twining [12, extended to 3D by VesterChristensen et al. [13, using the distance and cost functions of [8]. A steepest descent algorithm implemented as the inverse compositional algorithm by Baker and Matthews [14 reduces the registration time to 4-8 minutes per shape registered on a 1.7 GHz laptop PC. From the Procrustes [15], registered shapes a shape model and a deformation model are created. Generally, the impressions do not depict the exact same part of the ear, only an overlapping region; hence, the models are cropped to their common subset.

\section{Analysis and Results}

Visual inspection of the data reveals that almost all of the subjects have a clear visible deformation of their ear canal due to movement of the mandible. It is evident that a deformation occurs in the canal that has its maximum on the anterior side of the wall between the first and the second bend. As seen from fig. 3 the mean deformation is exactly that. This confirms our observations from the histological sectioning. Additionally, a deformation of Tragus, AntiTragus and Cavum Concha can be observed in the deformation model, which again corresponds well with the observations from the histological sectioning. The magnitude of the deformation varies among individuals from $\approx 0.2 \mathrm{~mm}$. to $2.3 \mathrm{~mm}$. Using the mean shape as reference the average, minimum and maximum 

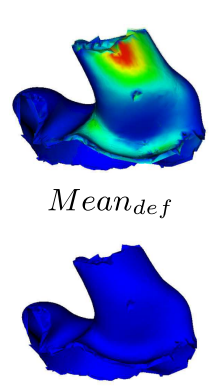

Mean shape

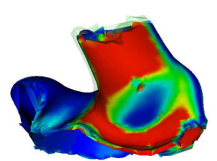

$P C 1_{\text {def }}$

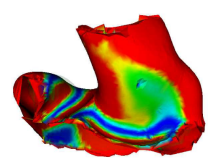

$P C 1_{\text {shape }}$

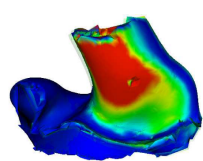

$P C 2_{\text {def }}$

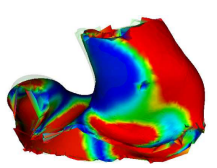

$P C 2$ shape

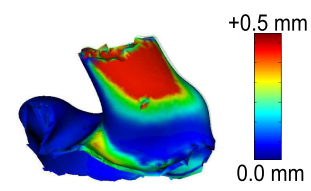

$P C 3_{\text {def }}$

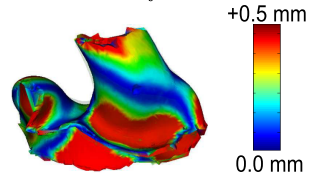

$P C 3_{\text {shape }}$

Fig. 3. The mean deformation and the first 3 modes of deformation variation and the mean shape and the first 3 modes of shape variation. All +2 standard deviations.

deformation over all sets of impressions can be calculated as average $=0.4349$ mm. $\min \approx 0.0 \mathrm{~mm}$. and $\max \approx 2.3 \mathrm{~mm}$..

It is a well known fact that ear size and gender are related [7. It is observed that the first mode of variation $\left(P C 1_{\text {shape }}\right)$ of our shape model is related to size (fig. 3). This is confirmed by using a logistic regression model to predict gender from this variable. Let $p$ be the posterior probability of a male. Then the model $\operatorname{logit}(p)=\alpha+\beta P C 1_{\text {shape }}$ is significant with significance levels less than $7 \%$. Visual inspection of the deformation fields has led us to suspect that males tend to have larger deformations than females. Hence, a logistic regression was performed with the mean amount of deformation over the entire shape as predictor of the gender. A model without intercept was chosen since no deformation should model odds 50/50 between genders. The resulting model is significant at a $4 \%$ level, confirming the hypothesis. The modelled difference between genders in deformation is most likely related to the differences in the male and female mandible. Such discrimination has been reported by Giles [16] and Graw [17] using the size and strength of the mandible.

\subsection{Shape Related to Deformation}

To investigate if shape and deformation are related, the deformation and the shape models are investigated (see fig. 3). Comparison of the first 5 modes explaining $82 \%$ of the total variation of the deformation model and the first 7 modes explaining $80 \%$ of the total variation of the shape model are made. The number of modes are found using parallel analysis by Horn [18. The first mode of deformation variation, containing primarily size of the deformation, cannot be explained by any of the 7 modes from the shape model. However, the next 3 modes of variation can. The second mode of deformation can be interpreted as change in angle of the canal in the plane bisecting the coronal-transversal angle in relation to Concha. This mode can be modelled by the second and third 
mode from the shape model. These two modes represents the vertical length of the Concha and the angle of the canal in the transversal plane. The third mode of deformation is the angle of the canal in relation to Concha in the transversal plane and the shape of the canal; round or oval. This mode of deformation can be modelled by the 6 th mode of shape variation. Both these models $P C 2_{\text {def }}=$ $\alpha+\beta_{1} P C 2_{\text {shape }}+\beta_{2} P C 2_{\text {shape }}$, and $P C 3_{\text {def }}=\alpha+\beta_{1} P C 6_{\text {shape }}$ are significant at the $1 \%$ level. The 4 th deformation mode is the bending of the canal in the transversal plane and the deformation of the Intertragic notch and can be related to the roundness of Concha and the angle of the canal in the coronal plane. The model has the form, $P C 4_{\text {def }}=\alpha+\beta_{1} P C 4_{\text {shape }}$ and is significant at the $1 \%$ level. The first mode of shape variation mode can explain the 5 th mode of deformation variation with significance $p<0.08$. Combined with the 6 th mode the significance becomes $p<0.06$.

\subsection{Analysis of the Deformation Field}

Now we will examine the deformation field for an individual ear in more detail, i.e. at every vertex of the triangulated surface representing the ear we will test if a significant deformation occurs as a function of opening the mouth. This involves simultaneous testing of $\approx 10000$ hypotheses. In order to do this we will employ Efrons [19] procedure for estimating the empirical null hypothesis for each individual. Using a i.i.d. normal assumption for the deformation vector elements under the null hypothesis $-H_{i}$ - we have that the magnitude of the deformation vector for the $i$ th vertex $Y_{i}$ follow a $\sigma \chi(3)$ distribution. For each vertex we can transform the $Y_{i}$ 's to $z$-values ( $\Phi$ is the standard normal cumulative distribution), where

$$
z_{i}=\Phi^{-1}\left(\operatorname{prob}\left\{Y_{i}>y_{i}\right\}, \quad z_{i} \mid H_{i} \in N(0,1)\right.
$$

The latter part is the theoretical null hypothesis. In Fig. 4(a) we show as a bar plot the histogram of $z$ 's from an experiment where two impressions have been taken from the same ear with closed mouth. The heavy right tail was expected due to shifting of ear wax, hair etc. We approximate the histogram with a smoothing spline and extract the maximum point and the full width half maximum of the (first) major top. This provides us with robust estimates of the mean and standard deviation under the null hypothesis. The empirical null is $z_{i} \mid H_{i} \in N(-0.40,0.60)$. The reasons for the difference between the theoretical and empirical nulls may be hidden correlations or the presence of genuine but uninteresting small effects.

Assuming that for each ear a large proportion of the vertices will exhibit no change due to mouth opening we can use a similar procedure to identify those vertices where significant change occur. In Fig. 4(b-c) the null is estimated from the first major peak and $95 \%$ and $99 \%$ quantiles are determined. The corresponding vertices where significant changes occur are shown in Fig. 4(d-f). We see that significant changes occur in Cavum Concha, at Tragus and Anti-Tragus and in the canal; in full correspondence with our expectations and precisely 


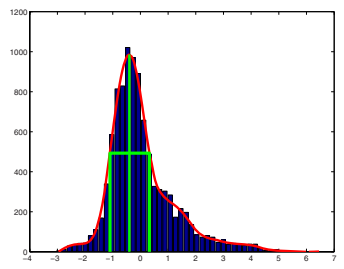

$a$

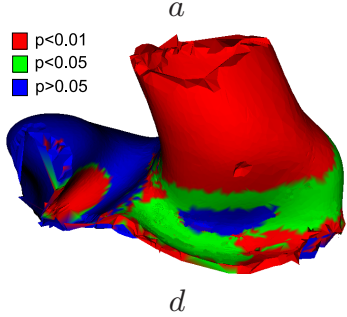

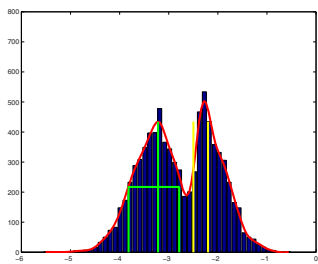

$b$

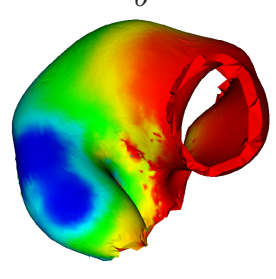

e
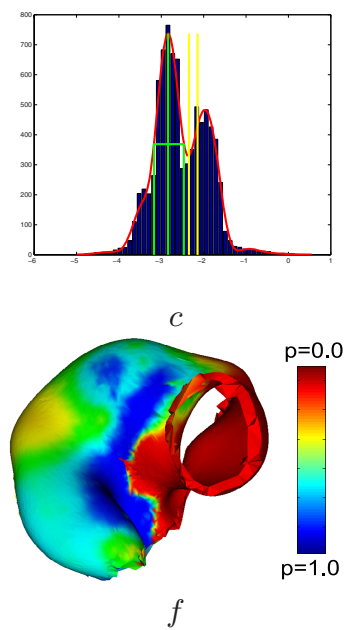

Fig. 4. (a) Histogram of the $z$-values under the null hypothesis and robust estimation of the normal parameters using a smoothing spline. (b-c) Histograms of $z$-values, robust null estimation and $95 \%$ and $99 \%$ quantiles for two ears.(d) the $95 \%$ and $99 \%$ quantiles of the ear in b. (e-f) p-value maps of b and c respectively.

where most hearing aids are situated in the ear. This confirms that movement of the mandible causes discomfort for some hearing-aid users.

\section{Conclusion}

We have shown that it is possible to consistently locate regions of significant deformation caused by movement of the mandible in all subjects. The occurrence of the deformations corresponds well to the physiology of the human ear, in terms of soft tissue and bony structures. The locations that are deforming the most are exactly where hearing aids normally are situated in the ear. Hence, we can confirm that movement of the mandible can cause discomfort in the ear when wearing a hearing aid. Furthermore, we have shown that males in general are more prone to deformation of the ear and canal and that the common assumption of men having larger ears than women seems to hold. Finally we have shown several significant relations between the shape of the ear and canal and the deformation occurring during movement of the mandible. We can explain $50 \%$ of the variation of the deformation using the first 6 modes of variation from the shape model. Our findings are very significant, even when considering the limited size of the data set. The features described by the modes of variation in the shape model can be used as guidelines to detect potentially problematic cases. They point to a problem caused by a specific kind of deformation, thus enabling the dispenser or hearing-aid manufacturer to take appropriate actions to eliminate the problem. 


\section{References}

1. Kochkin, S.: MarkeTrak V: "Why my hearing aids are in the drawer": The consumers perspective. The Hearing Journal 53(2), 34-39 (2000)

2. Sorensen, M.S., Dobrzeniecki, A.B., Larsen, P., Frisch, T., Sporring, J., Darvann, T.A.: The visible ear: A digital image library of the temporal bone. ORL 64, 378381 (2002)

3. Oliviera, R., Hammer, B., Stillman, A., Holm, J., Jons, C., Margolis, R.: A look at ear canal changes with jaw motion. Ear and Hearing 13(6), 464-466 (1992)

4. Oliviera, R., Babcock, M., Hoeker, M.V.G., Parish, B.: The dynamic ear canal and its implications: The problem may be the ear, and not the impression. Hear Reviews 12(2), 18-19 (2005)

5. Grenness, M.J., Osborn, J., Weller, W.L.: Mapping ear canal movement using areabased surface matching. JASA 111(2), 960-971 (2002)

6. Pirzanski, C.: Despite new digital technologies, shell modelers shoot in the dark. The Hearing Journal 59(10), 28-31 (2006)

7. Paulsen, R.R., Larsen, R., Laugesen, S., Nielsen, C., Ersbøll, B.K.: Building and testing a statistical shape model of the human ear canal. In: Dohi, T., Kikinis, R. (eds.) MICCAI 2002. LNCS, vol. 2488, Springer, Heidelberg (2002)

8. Darkner, S., Vester-Christensen, M., Larsen, R., Paulsen, R.R., Nielsen, C.: Automated 3D rigid registration of open 2D manifolds. In: Larsen, R., Nielsen, M., Sporring, J. (eds.) MICCAI 2006. LNCS, vol. 4190, Springer, Heidelberg (2006)

9. Darkner, S., Vester-Christensen, M., Larsen, R., Paulsen, R.R.: Evaluating a method for rigid registration. In: SPIE Medical Imaging 2007 (February 2007)

10. Bærentzen, J., Aanæs, H.: Signed distance computation using the angle weighted pseudo-normal. IEEE Transactions on Visualization and Computer Graphics 11(3), 243-253 (2005)

11. Whitted, T.: An improved illumination model for shaded display. Commun. ACM 23(6), 343-349 (1980)

12. Cootes, T., Marsland, S., Twining, C., Smith, K., Taylor, C.: Groupwise diffeomorphic non-rigid registration for automatic model building, vol. IV, pp. 316-327 (2004)

13. Vester-Christensen, M., Erbou, S.G., Darkner, S., Larsen, R.: Accelerated 3D image registration. In: SPIE Medical Imaging 2007 (February 2007)

14. Baker, S., Matthews, I.: Lucas-Kanade 20 years on: A unifying framework. International Journal of Computer Vision 56(3), 221-255 (2004)

15. Dryden, I.L., Mardia, K.: Statistical Shape Analysis. Wiley, Chichester (1998)

16. Giles, E.: Sex determination by discriminant function analysis of the mandible. American Journal of Physical Anthropology 22(2), 129-135 (1964)

17. Graw, M.: Significance of the classical morphological criteria for identifying gender using recent skulls, vol. 3 (January 2001)

18. Horn, J.L.: A rationale and test for the number of factors in factor analysis. Psychometrika 30, 179-185 (1965)

19. Efron, B.: Large-scale simultaneous hypothesis testing: the choice of a null hypothesis. Journal of the American Statistical Association 99, 96-104 